ARTICLE

DOI: $10.1038 / s 41467-018-06374-z$

\title{
Selective prebiotic conversion of pyrimidine and purine anhydronucleosides into Watson-Crick base-pairing arabino-furanosyl nucleosides in water
}

Samuel J. Roberts' ${ }^{1}$, Rafał Szabla ${ }^{2,3}$, Zoe R. Todd ${ }^{4}$, Shaun Stairs ${ }^{1}$, Dejan-Krešimir Bučar ${ }^{1}$, Jiři Šponer ${ }^{3}$, Dimitar D. Sasselov ${ }^{4} \&$ Matthew W. Powner (iD ${ }^{1}$

Prebiotic nucleotide synthesis is crucial to understanding the origins of life on Earth. There are numerous candidates for life's first nucleic acid, however, currently no prebiotic method to selectively and concurrently synthesise the canonical Watson-Crick base-pairing pyrimidine $(C, U)$ and purine $(A, G)$ nucleosides exists for any genetic polymer. Here, we demonstrate the divergent prebiotic synthesis of arabinonucleic acid (ANA) nucleosides. The complete set of canonical nucleosides is delivered from one reaction sequence, with regiospecific glycosidation and complete furanosyl selectivity. We observe photochemical 8mercaptopurine reduction is efficient for the canonical purines $(A, G)$, but not the noncanonical purine inosine (I). Our results demonstrate that synthesis of ANA may have been facile under conditions that comply with plausible geochemical environments on early Earth and, given that ANA is capable of encoding RNA/DNA compatible information and evolving to yield catalytic ANA-zymes, ANA may have played a critical role during the origins of life.

\footnotetext{
${ }^{1}$ Department of Chemistry, University College London, 20 Gordon Street, London WC1H OAJ, UK. ${ }^{2}$ Institute of Physics, Polish Academy of Sciences, Al. Lotników 32/46, PL-02668 Warsaw, Poland. ${ }^{3}$ Institute of Biophysics of the Czech Academy of Sciences, Královopolská 135, 61265 Brno, Czech Republic. ${ }^{4}$ Harvard-Smithsonian Center for Astrophysics, Department of Astronomy, Harvard University, 60 Garden Street, Cambridge, MA 02138, USA.

Correspondence and requests for materials should be addressed to M.W.P. (email: matthew.powner@ucl.ac.uk)
} 
T he synthesis of the complete set of canonical Watson-Crick base-pairing nucleosides [adenosine (A), cytidine $(\mathrm{C})$, guanosine $(\mathrm{G})$ and uridine $(\mathrm{U})$ ] under conditions that do not violate the accepted plausible geochemical environments on early Earth is an essential step towards elucidating the origins of life on Earth ${ }^{1-3}$. However, while many 'plausible' nucleoside candidates have been suggested to have played a role at the origins of life $\mathrm{e}^{4-6}$, the concurrent prebiotic synthesis of a complete set of nucleoside monomers remains an unresolved challenge for any of the proposed genetic polymers [e.g., ribonucleic acid (RNA), arabinonucleic acid (ANA), threonucleic acid (TNA) and pyranosyl-ribonucleic acid (pRNA) $]^{3,5,7-24}$. Accordingly, we set out to elucidate chemical reactions that could address this problem. We have previously reported a prebiotic synthesis of pyrimidine ribonucleotides 1C and $1 \mathbf{U}^{16}$. More recently, we reported the divergent synthesis of 1C, 1U and 8-oxo-purine ribonucleotides $2 \mathrm{~A}$ and $\mathbf{2 I}$ (Fig. 1, red arrows $)^{22}$. However, no divergent prebiotic synthesis of pyrimidine and purine nucleoside monomers bearing the canonical Watson-Crick base-pairing nucleobases has yet been elucidated $^{7,21-23}$.

ANA displays many properties that make it an attractive candidate for the first genetic polymer of life. ANAs can equilibrate between helix and stem-loop structures, which mimic DNA and RNA, respectively ${ }^{25}$. ANA can form a complementary Watson-Crick base-paired duplex with $\mathrm{RNA}^{26,27}$, and can be readily transcribed (from DNA) and reverse transcribed (to DNA $)^{28}$. Additionally, Holliger and co-workers recently evolved catalytic ANA-zymes that can achieve RNA phosphodiester cleavage ${ }^{6}$. Notably, the ANA phosphodiester backbone is also far more resistant to hydrolysis than its RNA analogue ${ }^{26}$.

Anhydrocytidine (3C), a key intermediate in our previously reported prebiotic pyrimidine synthesis ${ }^{16}$, undergoes hydrolysis at near neutral $\mathrm{pH}(\geq 6.5)^{22}$ to quantitatively afford arabinocytidine (ara-4C; Fig. 1, blue arrow). This facile hydrolysis suggests that a simple prebiotic synthesis of arabino-nucleotides may be achievable. Importantly, the synthesis of a complete set of arabinosides requires differential reactivity between the purine and pyrimidine precursors $\mathbf{3 A} / \mathbf{3 G}$ and $\mathbf{3 C}$ (Fig. 1), respectively ${ }^{16,22}$. Pyrimidine $\boldsymbol{a r a}-4 \mathrm{C}$ can be accessed by direct hydrolysis of $\mathbf{3 C}$, whereas hydrolysis of $\mathbf{3 A}$ and $\mathbf{3 G}$ would furnish 8-oxo-purines (8-oxo-4A and 8-oxo-4G; Fig. 1, dashed arrow) ${ }^{22,29}$ rather than the desired purine nucleosides $\boldsymbol{a r a} \mathbf{- 4 A}$ and $\boldsymbol{a r a}-\mathbf{4 G}$. Therefore, it is of note that purine precursors $\mathbf{3} \mathbf{A}^{22}, \mathbf{3 I}$ and $\mathbf{3 G}$ are highly resistant to alkaline hydrolysis, even in extremely alkaline $(\mathrm{pH}>12)$ solutions. Accordingly, we viewed this subtle, yet pronounced, difference in reactivity as an ideal source for chemical differentiation that could be exploited while building the canonical nucleobases on a preformed furanosylsugar scaffold. We envisaged sulfur-a critical element in the development of divergent ribonucleoside syntheses ${ }^{22}$, with widespread use in prebiotic chemistry $22,30-35$ - would hold the key to site-selective purine reduction.

Here, we demonstrate a divergent route to synthesise a complete set of canonical (A, G, C and U) nucleosides from one plausibly prebiotic reaction sequence. Interestingly, photochemical reduction of (intermediate) 8-mercaptopurines is observed to be highly efficient for the desired canonical purines (A and G), but not the non-canonical purine inosine (I). The facile prebiotic synthesis of ANA indicates that it may have played an important role during the origins of life, and the selective photochemical reduction of 8-mercaptopurines provides a physical mechanism for prebiotic nucleobase selection en route to the Watson-Crick base-pairing nucleosides.

\section{Results}

8-Mercaptopurine synthesis. We suspected that addition of hydrogen sulfide $\left(\mathrm{H}_{2} \mathrm{~S}\right)$ to $\mathbf{3 A}, \mathbf{3 G}$ and $\mathbf{3 I}$ in water would selectively introduce sulfur at the $\mathrm{C} 8$-carbon atom, and consequently direct regiospecific reduction of the canonical purine nucleobases on the preformed sugar scaffold (Fig. 1, blue arrows). Previously, Ikehara and Ogiso established that $\mathbf{3 A}$ reacts with liquid $\mathrm{H}_{2} \mathrm{~S}$ in pyridine at $100^{\circ} \mathrm{C}$ (sealed in a steel tube) to afford 8-mercaptoarabino-adenosine $(\boldsymbol{a r a - 5 A})^{36}$. However, 3A, 3G and 3I were remarkably stable to alkaline hydrolysis (Supplementary Figs. 1 and 2). Therefore, we began our investigation by exploring the mild, plausibly prebiotic, aqueous thiolysis of the prebiotic purine



Fig. 1 Divergent prebiotic nucleotides synthesis. Red arrows: Previous work; a prebiotic pathway to cytidine--2', $3^{\prime}$-cyclic phosphate (1C), uridine-2', $3^{\prime}$-cyclic phosphate (1U), 8-oxo-adenosine-2', $3^{\prime}$-cyclic phosphate (2A) and 8-oxo-inosine-2', $3^{\prime}$-cyclic phosphate (21) ${ }^{16,22}$. Dashed arrow: Hydrolysis of 8,2'anhydropurines (3A, $\mathbf{3 I}$ and $\mathbf{3 G}$ ) is not observed, which provides chemical differentiation from 2,2'-anhydropyrimidine (3C) that readily hydrolyses to $\beta$-arabino-adenosine (ara-4C). Blue arrows: This work; a prebiotic pathway to $\beta$-arabino-cytidine (ara-4C), $\beta$-arabino-uridine (ara-4U), $\beta$-arabino-adenosine (ara-4A), $\beta$-arabino-inosine (ara-4I) and $\beta$-arabino-guanosine (ara-4G) 



Fig. 2 Prebiotic synthesis of arabino-adenosine (ara-4A). ${ }^{1} \mathrm{H}$ NMR spectra $\left(600 \mathrm{MHz}, 9: 1 \mathrm{H}_{2} \mathrm{O} / \mathrm{D}_{2} \mathrm{O}, 25^{\circ} \mathrm{C}, \delta=5.5-9.0\right.$ ppm) showing: a anhydroadenosine (3A; red). b Crude 8-mercapto-arabino-adenosine (ara-5A; blue) observed upon incubation of anhydroadenosine $(\mathbf{3 A} ; 35.7 \mathrm{mM})$ and $\mathrm{H}_{2} \mathrm{~S}\left(670 \mathrm{mM}, 60{ }^{\circ} \mathrm{C}\right.$, $7 \mathrm{~d}, \mathrm{pH} 7)$. c Crude arabino-adenosine (ara-4A; purple) after irradiation ( $\lambda=300 \mathrm{~nm}$ ) of 8 -mercapto-arabino-adenosine (ara-5A; $2 \mathrm{mM}$, room temperature, $16 \mathrm{~h}, \mathrm{pH} 7)$

\section{Table 1 Percentage conversions for nucleoside reactions}

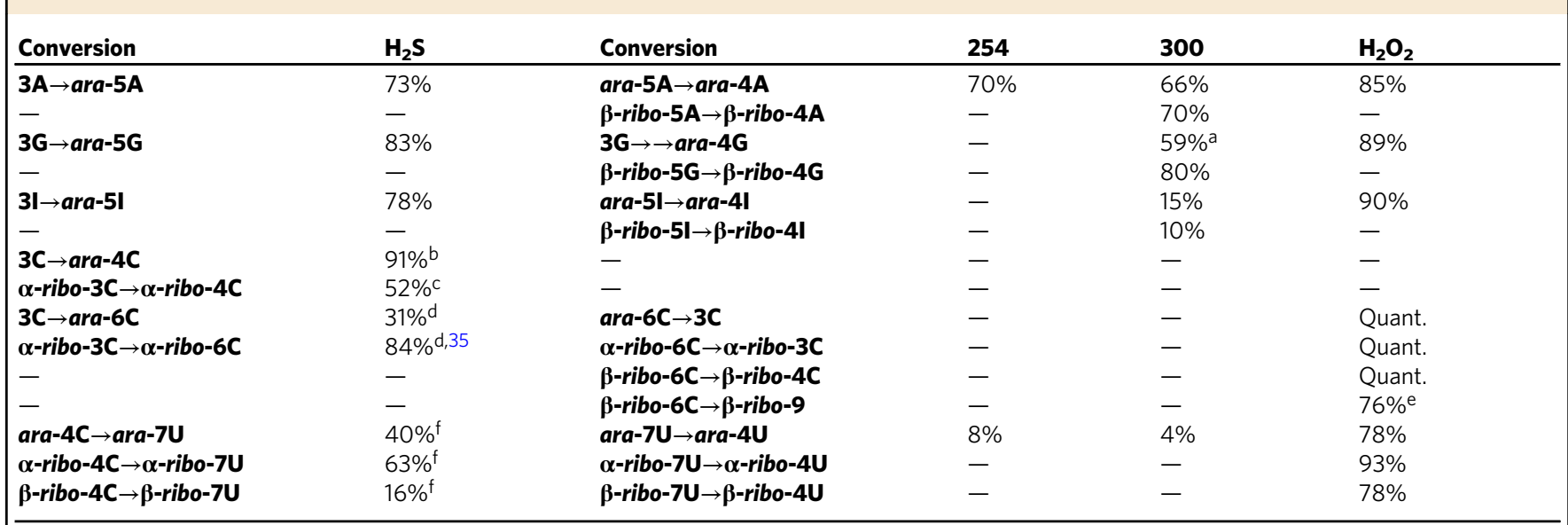

Conversions were directly determined by ${ }^{1} \mathrm{H} \mathrm{NMR}(600 \mathrm{MHz})$ spectroscopy in the crude product mixture. Conversion observed upon: $\mathrm{H}_{2} \mathrm{~S}$ : reaction with $\mathrm{H}_{2} \mathrm{~S}\left(20\right.$ equiv.) in water ( $\mathrm{pH} 7,60^{\circ} \mathrm{C}, 7 \mathrm{~d}$ ); 254: irradiation at $\lambda=254 \mathrm{~nm}$ in water $\left(38^{\circ} \mathrm{C}, \mathrm{pH} 6.5,16 \mathrm{~h}\right) ; 300$ : irradiation at $\lambda=300 \mathrm{~nm}$ in water $\left(38^{\circ} \mathrm{C}, \mathrm{pH} 6.5,16 \mathrm{~h}\right.$ ); $\mathrm{H}_{2} \mathrm{O}_{2}$ : reaction with $\mathrm{H}_{2} \mathrm{O}_{2}(3$ equiv.) in water ( $\mathrm{pH} 7$, room temperature) a $22 \mathrm{~h}$ irradiation

${ }^{b}$ Conversion at room temperature. Conversion of ara-4C to ara-7U occurs at $60^{\circ} \mathrm{C}$ over $7 \mathrm{~d}$ to afford a mixture of ara-4C/ara-7U (1.5:1) in $95 \%$ combined conversion

CUnoptimised hydrolysis observed after 7 d incubation with $\mathrm{H}_{2} \mathrm{~S}\left(20\right.$ equiv.) in water $\left(60^{\circ} \mathrm{C}, \mathrm{pH} 7\right)$, observed alongside $39 \%$ hydrolysis of $\boldsymbol{\alpha}$-ribo-4C to $\boldsymbol{\alpha}$-ribo-7U ( $91 \%$ combined $\boldsymbol{\alpha}$-ribo-4C $+\boldsymbol{\alpha}$-ribo7U)

dConversion in formamide with $\mathrm{H}_{2} \mathrm{~S}$ (4 equiv.)

eBuffered at pH 3 with glycine; $\boldsymbol{\beta}$-ribo-9 observed alongside partial hydrolysis to $\boldsymbol{\beta}$-ribo-4C (24\%)

fUnoptimised thiolysis observed after $7 \mathrm{~d}$ incubation with $\mathrm{H}_{2} \mathrm{~S}$ (20 equiv.) at $60^{\circ} \mathrm{C}$ and pH 7 to investigate comparative rates of cytidine $\mathrm{C} 4$-thiolysis. Residual starting material ara-4C (52\%), $\boldsymbol{\alpha}$-ribo-4C $(37 \%)$ and $\boldsymbol{\beta}$-ribo-4C $(84 \%)$, respectively, accounted $(>90 \%)$ for the residual mass balance

precursor $\mathbf{3} \mathbf{A}^{22,33,35}$. Incubation of $\mathrm{H}_{2} \mathrm{~S}(670 \mathrm{mM})$ with $\mathbf{3 A}(35.7$ $\mathrm{mM})$ in water $\left(\mathrm{pH} 7,60^{\circ} \mathrm{C}, 7 \mathrm{~d}\right)$ afforded remarkably clean conversion of $\mathbf{3 A}$ to $\boldsymbol{a r a - 5 A}$ (73\%; Fig. 2, Table 1 and Supplementary Fig. 3).

Intrigued by the remarkably clean, high-conversion thiolysis of 3A, we next investigated the thiolysis ( $\mathrm{pH} \mathrm{7,60}{ }^{\circ} \mathrm{C}, 7 \mathrm{~d}$ ) of $\mathbf{3 G}$ and 3I. We observed ara-5G (83\%) and ara-5I (78\%; 71\% isolated yield after $5 \mathrm{~d}$ ) (Table 1 and Supplementary Figs. 4 and 5). Next, 1:1 $\mathbf{3 A} / \mathbf{3 I}$ was subjected to thiolysis and gave a high conversion to both ara-5A (66\%) and ara-5I (75\%) (Supplementary Fig. 6).
Even under these mild, aqueous conditions, highly efficient thiolysis of $\mathbf{3 A}, \mathbf{3 G}$ and $\mathbf{3 I}$ was observed, demonstrating that sulfur can be regioselectively introduced to the C8-carbon atom of purines under plausibly prebiotic conditions.

Photochemical purine reduction. For purine reduction, we initially investigated the effect of UV light on 8-mercaptopurines 5, because the atmosphere of the early Earth lacked an ozone layer ${ }^{37}$, allowing UV light $(\lambda>204 \mathrm{~nm})$ to irradiate Earth's surface ${ }^{38,39}$. We envisaged that UV irradiation of ara-5A would lead 
to $\pi-\pi^{*}$ excitation, followed by $\mathrm{C}-\mathrm{S}$ bond fragmentation to afford an N-heterocyclic carbene tautomer of ara-4A. Pleasingly, when ara-5A $(2.00 \mathrm{mM}, \mathrm{pH} 7)$ was irradiated $(\lambda=300$ or $254 \mathrm{~nm})$ in water we observed extremely clean conversion to ara-4A in $66 \%$ and $70 \%$, respectively, after $16 \mathrm{~h}$ (Fig. 2 and Supplementary Figs. 7-9). Notably, ara-4A was the only nucleoside product observed after irradiation. Irradiation $(\lambda=300 \mathrm{~nm})$ of the inosine analogue ara-5I gave arabino-inosine ara-4I (Supplementary Fig. 10). However, upon complete consumption of ara-5I, the observed conversion to ara-4I (15\%) was significantly lower than for $\boldsymbol{a r a - 4 A}(66 \%)$ (Table 1). Next, the two-step thiolysis/irradiation sequence was investigated for 3G. Anhydronucleoside 3G $(5.85 \mathrm{mM})$ was thiolysed $\left(\mathrm{H}_{2} \mathrm{~S}, \mathrm{pH} 7,60^{\circ} \mathrm{C}, 7 \mathrm{~d}\right)$ and then irradiated $(\lambda=300 \mathrm{~nm})$. The reaction was monitored until complete consumption of the intermediate (ara-5G) was observed (Supplementary Fig. 11). Once again, we observed a good conversion (59\% after $22 \mathrm{~h}$; Table 1 ) to the desired product arabino-guanosine (ara-4G). Irradiation of 1:1 ara-5A/ara-5I confirmed the disparity between the conversions observed for the reduction to yield the canonical nucleobases A and $\mathrm{G}$ and wobble base-pairing I: $62 \%$ and $13 \%$ conversion to $\boldsymbol{a r a}-4 \mathrm{~A}$ and $\boldsymbol{a r a}-\mathbf{4 I}$, respectively, was observed (Supplementary Fig. 12). Notably, the irradiation $(\lambda=300$ or $254 \mathrm{~nm}$ ) of $1: 1$ ara-4A/ara-4I (Supplementary Fig. 13) demonstrated equal product stability with respect to UV irradiation (e.g., $73 \%$ each after $16 \mathrm{~h}$ at $\lambda=300 \mathrm{~nm}$ ), and therefore did not account for the intriguing differential percentage conversion observed between the canonical (A and G) and noncanonical (I) 8-mercapto-nucleosides. Subsequently, we irradiated the ribo-mercaptopurines ribo-5A, ribo-5G and ribo-5I, and observed highly efficient photo-reduction of the $A$ and $G$ ribonucleosides, but poor yielding reduction of the I ribo-nucleoside (Table 1 and Supplementary Figs. 14-16).

Quantum chemical studies. The observed difference in the photo-reduction of $\mathbf{5 A}$ and $\mathbf{5 G}$ compared to $\mathbf{5 I}$ cannot be directly connected to any remarkable differences in their UV-absorption features (Supplementary Figs. 117-119). However, quantum chemical studies $[\mathrm{ADC}(2)]^{40,41}$ and femtosecond transient absorption spectroscopy (FTAS) suggest that after initial UV excitation these purines undergo different singlet-to-triplet decay pathways.

UV excitation of mercaptopurines (5) was calculated to result in the population of low-lying excited singlet states $\left(\pi \pi_{\mathrm{CS}^{*}}\right.$, $\pi \pi_{\mathrm{RING}^{*}}{ }^{*}$ and $n \pi_{\mathrm{CS}}{ }^{*}$ ), which all exhibit significant spin-orbit coupling (SOC) with the triplet manifold (Supplementary Table 2 and Supplementary Discussion). Therefore, these singlet states are expected to undergo efficient intersystem crossing (ISC) to populate the triplet excited states of 5, as noted for other thionucleosides and thionucleobases ${ }^{42-45}$. Two important triplet minima were identified in our calculations (Fig. 3). First, the $\mathrm{T}_{1}\left({ }^{3} \pi \pi_{\mathrm{CS}}{ }^{*}\right)$ triplet minimum (Fig. 3a), which leads to C8thiocarbonyl elongation $(\approx 0.1 \AA)$ and localisation of unpaired electrons on the $s p^{3}$-hybridised C8-carbon and sulfur atoms. Second, the $\mathrm{T}_{1}\left({ }^{3} \pi \pi_{\mathrm{RING}}{ }^{\star}\right)$ triplet minimum (Fig. 3a), which leads to pyrimidine ring puckering and localisation of unpaired electrons on the partially $s p^{3}$-hybridised C2- and C5-carbon atoms. The $\boldsymbol{a r a}-\mathbf{5} \mathbf{A}^{3} \pi \pi_{\text {ring }}{ }^{*}$ triplet is significantly higher in energy than the ara-5A ${ }^{3} \pi \pi_{\mathrm{CS}}{ }^{*}$ triplet; consequently, population of the ${ }^{3} \pi \pi_{\mathrm{CS}}{ }^{\star}$ triplet is predicted to be the dominant pathway during ISC following UV excitation of ara-5A (Fig. 3d). Accordingly, we expect that C8-photo-reduction is triggered in the ${ }^{3} \pi \pi_{\mathrm{CS}}{ }^{*}$ triplet

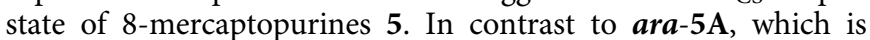
expected to only populate the ${ }^{3} \pi \pi_{\mathrm{CS}}{ }^{*}$ triplet state, ara-5I and $\boldsymbol{a r a}$ 5G are predicted to populate both triplet states $\left({ }^{3} \pi \pi_{C S^{*}}\right.$ and $\left.{ }^{3} \pi \pi_{\text {ring }}{ }^{*}\right)$. Optimisation of the mercaptopurine excited state geometries indicated that the $\mathrm{T}_{1}$ hypersurface of ara-5I (Fig. 3c) and ara-5G (Supplementary Fig. 51) are similar in topography to those reported for thiopyrimidine nucleobases ${ }^{46}$. Consequently, we expected $\boldsymbol{a r a - 5 I}$ and $\boldsymbol{a r a - 5 G}$ to exhibit similar UV excitation behaviour to thiopyrimidines, which are observed to undergo two competing excited state decay pathways ${ }^{46}$.

Short excited state lifetimes are expected for the redox active $\mathrm{T}_{1}\left({ }^{3} \pi \pi_{\mathrm{CS}}{ }^{*}\right)$ triplet states, due to their very large SOC values (ara5A: $99.5 \mathrm{~cm}^{-1}$; ara-5I: $\left.81.4 \mathrm{~cm}^{-1}\right)$ and low $(0.23 \mathrm{eV})$ statecrossing barrier to the electronic ground state $\left(\mathrm{S}_{0}\right)$. Conversely, the ara-5I $\mathrm{T}_{1}\left({ }^{3} \pi \pi_{\mathrm{RING}}{ }^{*}\right)$ triplet state exhibits a very low SOC value $\left(1.44 \mathrm{~cm}^{-1}\right)$ and is predicted to be much longer lived. Moreover, the ara-5I T ${ }_{1}\left({ }^{3} \pi \pi_{\mathrm{RING}}{ }^{*}\right)$ triplet minimum is estimated to be $0.2 \mathrm{eV}$ lower in energy than the redox active ara-5I $\mathrm{T}_{1}\left({ }^{3} \pi \pi_{\mathrm{CS}}{ }^{*}\right)$ triplet minimum, and predominant population of the non-redox active $\mathrm{T}_{1}\left({ }^{3} \pi \pi_{\mathrm{RING}}{ }^{*}\right)$ triplet minimum is expected following UV excitation of ara-5I because these two states are only separated by a moderate $(0.55 \mathrm{eV})$ energy barrier.

Upon first inspection it might seem surprising that, like ara-5I, both triplet states can be populated in ara-5G given its observed efficient photo-reduction. However, the ara-5G $\mathrm{T}_{1}\left({ }^{3} \pi \pi_{\mathrm{RING}}{ }^{*}\right)$ and $\mathrm{T}_{1}\left({ }^{3} \pi \pi_{\mathrm{CS}}{ }^{\star}\right)$ triplet minima are calculated to be nearly isoenergetic, and therefore these minima are expected to easily interconvert. Accordingly, efficient photo-reduction of ara-5G is thought to occur by continual repopulation of the redox active ara-5G $\mathrm{T}_{1}\left({ }^{3} \pi \pi_{\mathrm{CS}}{ }^{*}\right)$ triplet minimum.

Femtosecond transient absorption spectroscopy. We next sought to verify our theoretical predictions by FTAS for ara-5I (Fig. 3e) and ara-5A (Fig. 3f). The absorbance $(\lambda=380-450 \mathrm{~nm})$ observed in the first picosecond following ara-5A excitation matches the position and structure of the excited state absorption (ESA) spectrum simulated from the $S_{1}\left({ }^{1} \pi \pi_{C S}{ }^{*}\right)$ singlet minimum of ara-5A (Fig. 3f, inset). Initial singlet $\left({ }^{1} \pi \pi_{\mathrm{CS}}{ }^{\star}\right)$ state population following photo-excitation is also consistent with our calculated vertical excitation energies (Supplementary Table 1), which indicate that $\lambda=290-300 \mathrm{~nm}$ excitation would primarily populate this state (Fig. 3e). At probe time delays $>2$ ps after incident excitation of $\boldsymbol{a r a}-\mathbf{5 A}$, the absorbance evolved to cover a broader range of probe wavelengths, and the FTAS spectrum exhibits two characteristic features near $\lambda=360$ and $480 \mathrm{~nm}$. Although these features are partly covered by the bleaching bands, they match the simulated ara-5A $\mathrm{T}_{1}\left({ }^{3} \pi \pi_{\mathrm{CS}}{ }^{*}\right)$ triplet state ESA spectrum (Fig. $3 \mathrm{f}$, inset), which indicates population of this triplet state. The observed excited state lifetime of $\boldsymbol{a r a}-\mathbf{5 A}(\approx 70 \mathrm{~ns}$ at $\lambda=510 \mathrm{~nm})$ is also consistent with the predicted efficient photo-relaxation of this triplet state, which is characterised by high SOC with the electronic ground state.

Although excitation at $\lambda=290 \mathrm{~nm}$ could potentially populate both ara-5I singlet states (i.e., ${ }^{1} \pi \pi_{\mathrm{CS}}{ }^{*}$ and ${ }^{1} \pi \pi_{\text {ring }}{ }^{*}$ ), we did not observe any features in the FTAS spectrum that would correspond to the ${ }^{1} \pi \pi_{\mathrm{CS}}{ }^{\star}$ singlet state. No significant change in absorbance was recorded during the first 0.4 ps (Fig. 3e). We were unable to simulate the ESA spectrum for the ara-5I $S_{1}\left({ }^{1} \pi \pi_{\text {ring }}{ }^{*}\right)$ singlet state (its minimum-energy geometry coincided with the $\mathrm{S}_{1} / \mathrm{S}_{0}$ conical intersection), but we anticipate that population of this singlet state dominates the $0.4 \mathrm{ps}$ immediately after ara-5I excitation. The emergence of a sharp band at $\lambda=350 \mathrm{~nm}$ between 0.6 and $1.0 \mathrm{ps}$ was assigned to population of the redox active $\mathrm{T}_{1}\left({ }^{3} \pi \pi_{\mathrm{CS}}{ }^{*}\right)$ triplet state, which could be efficiently accessed from the initial $S_{1}\left({ }^{1} \pi \pi_{\text {ring }}{ }^{*}\right)$ singlet state because of the molecular orbital change associated with an $S_{1} \rightarrow T_{1}$ transition ${ }^{47}$. The redox active ara-5I $\mathrm{T}_{1}\left({ }^{3} \pi \pi_{\mathrm{CS}}{ }^{*}\right)$ triplet minimum is observed to be efficiently depopulated over the next 6 ps. The excited state population transfers to the lower-energy ara-5I $\mathrm{T}_{1}\left({ }^{3} \pi \pi_{\text {ring }}{ }^{*}\right)$ 
a

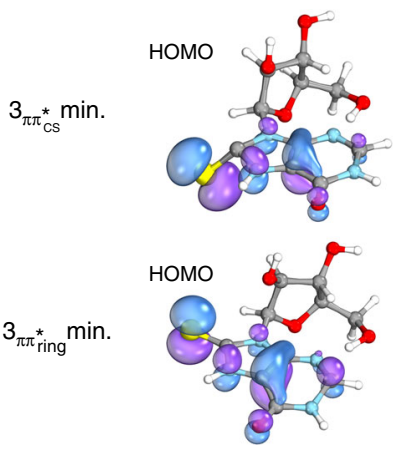

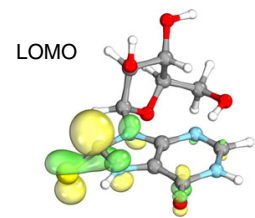

LOMO

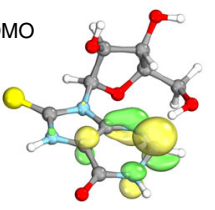

C

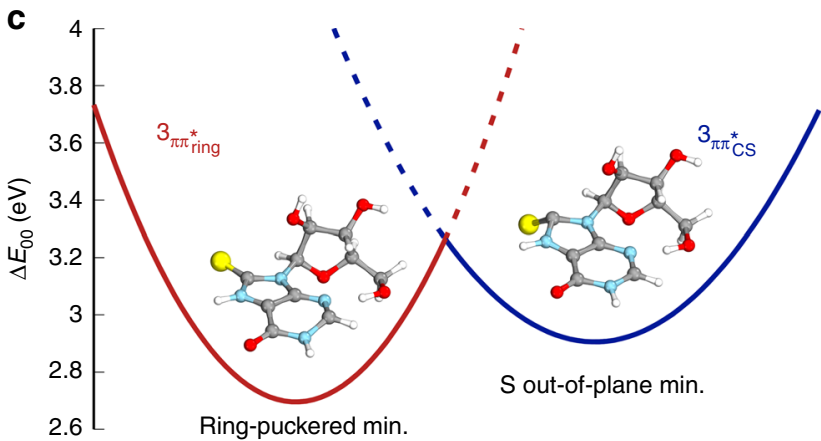

e



b

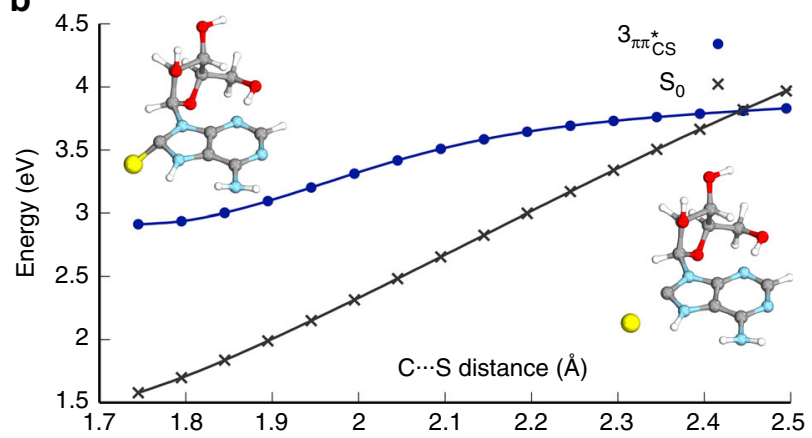

d

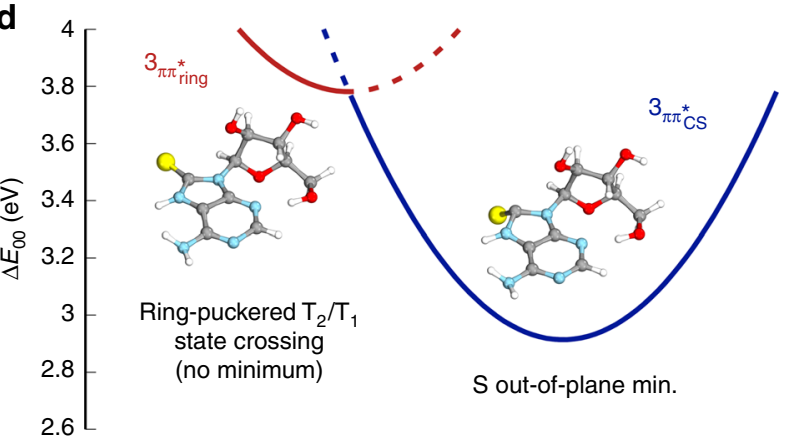

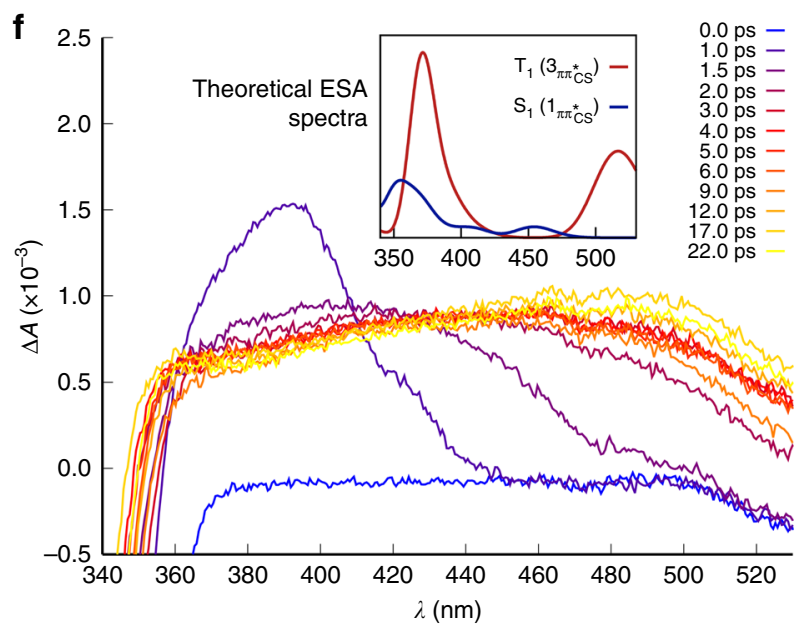

Fig. 3 Photochemical properties of $\boldsymbol{a r a - 5 I}$ and $\boldsymbol{a r a - 5 A}$. a Molecular orbitals for $T_{1}$ states $\left({ }^{3} \pi \pi_{C S}{ }^{\star}\right.$ and $\left.{ }^{3} \pi \pi_{\text {ring }}{ }^{\star}\right)$ of $\boldsymbol{a r a - 5 I}$. $\mathbf{b}$ Calculated potential energy profile of ara-5A $T_{1}{ }^{3} \pi \pi_{\mathrm{CS}}{ }^{*}$ state $\mathrm{C}-\mathrm{S}$ bond homolysis furnishing triplet sulfur atom and singlet C8-carbene tautomer of $\boldsymbol{a r a}-\mathbf{4 A}$. Blue line $=\mathrm{T}_{1}$-state energy, black line $=$ ground-state energy. The minimum-energy path along the C-S distance $(\AA)$ was obtained by calculation at the ADC(2)/cc-pVTZ level of theory. c Calculated ara-5I triplet excited state $T_{1}$ topography. Parabolas fitted to calculated $T_{1}$ minima energies and optimised $T_{2} / T_{1}$ minimum-energy state crossing, which represents the transition state between these minima. $\mathbf{d}$ Calculated ara-5A triplet excited state $\mathrm{T}_{1}$ topography. No ring-puckered minimum was found on the $\mathrm{T}_{1}$ hypersurface due to the higher ${ }^{3} \pi \pi_{\text {ring }}{ }^{*}$ state energy. e-f FTAS recorded between $\lambda=340$ and $530 \mathrm{~nm}$ with $\lambda=290 \mathrm{~nm}$ excitation (pump) pulses. e FTAS of ara-5I recorded between $\lambda=340$ and $530 \mathrm{~nm}$ with $\lambda=290 \mathrm{~nm}$ excitation (pump) pulses. Inset: Calculated excited state absorption spectra for the two possible configurations of ara-5I. f FTAS of ara-5A recorded between $\lambda=340$ and $530 \mathrm{~nm}$ with $\lambda=290 \mathrm{~nm}$ excitation (pump) pulses. Inset: Calculated excited state absorption spectra for the singlet and triplet states of ara-5A

triplet minimum (Fig. 3e), which is confirmed by the long excited state lifetime $(\approx 12 \mu \mathrm{s}$ at $\lambda=440 \mathrm{~nm})$ and the two characteristic bands $\left(\lambda_{\max }=350\right.$ and $\left.490 \mathrm{~nm}\right)$ in the ara-5I FTAS measurements, which are both consistent with the simulated ESA spectrum for the redox inactive triplet state (Fig. 3e, inset). The estimated excited state lifetime associated with the $\mathrm{T}_{1}\left({ }^{3} \pi \pi_{\text {ring }}{ }^{*}\right)$ triplet state is thought to be sufficient to enable bimolecular reactions and, consequently, the photochemical degradation pathways in ara-5I, which are not observed for ara-5A or ara5G. Photo-reduction appears to correlate directly with population of the $\mathrm{T}_{1}\left({ }^{3} \pi \pi_{\mathrm{CS}}{ }^{*}\right)$ triplet state for $\boldsymbol{a r a}-\mathbf{5} \mathbf{A}$, and we anticipate that the same triplet state would be responsible for photo-reduction in ara-5I and ara-5G. In particular, the observed short-lived population of the ara-5I $\mathrm{T}_{1}\left({ }^{3} \pi \pi_{\mathrm{CS}}{ }^{*}\right)$ triplet state $(0.6-7.0 \mathrm{ps})$ likely explains the poor photo-reduction observed for this nucleoside.

It appears probable that $\mathrm{C}-\mathrm{S}$ bond homolysis in ara-5A, initiated by population of the $\mathrm{T}_{1}\left({ }^{3} \pi \pi_{\mathrm{CS}}{ }^{*}\right)$ triplet minimum, would furnish the singlet C8-carbene tautomer of purine ara-4A (Fig. 3b). Our calculations indicate that $\mathrm{C}-\mathrm{S}$ bond homolysis requires $0.9 \mathrm{eV}$ to liberate the triplet sulfur atom. Excitation at $\lambda=300 \mathrm{~nm}$ would excite ara $\mathbf{5} \mathbf{A} \sim 1.2 \mathrm{eV}$ above the $\mathrm{T}_{1}\left({ }^{3} \pi \pi_{\mathrm{CS}}{ }^{*}\right)$ 
a<smiles>[Y]c1ccn([C@@H]2O[C@H](CO)[C@@H](O)[C@H]2O)c(=O)n1</smiles>

b<smiles>[Y]c1ccn([C@@H]2O[C@H](CO)[C@@H](O)[C@H]2O)c(=O)n1</smiles>

$\alpha-$ ribo-3C

$\alpha-$ ribo-4C

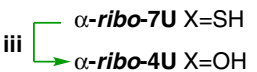

iv $\downarrow$ viii<smiles>Nc1ccn([C@@H]2O[C@H](CO)[C@@H](O)[C@H]2O)c(=S)n1</smiles>

$\alpha-$ ribo-6C

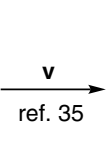

$\longleftarrow$<smiles>N=c1ccn([C@@H]2O[C@H](CO)[C@@H](O)[C@H]2O)cn1</smiles><smiles>N=c1ccn([C@@H]2O[C@H](CO)[C@@H](O)[C@H]2O)cn1</smiles><smiles>CC(C)(C)O[C@H]1[C@@H](O)[C@@H](CO)O[C@H]1n1ccc(N)nc1=S</smiles>

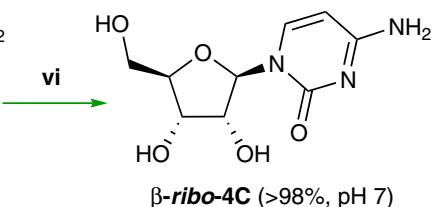<smiles>[Y][13C](C)(C)S(=O)(=O)O</smiles><smiles>[Y][Y]</smiles>

B-ribo-9 $(76 \%, \mathrm{pH} 3)$

$\mathrm{OH}$<smiles>[X]c1ccn([C@@H]2O[C@H](CO)[C@@H](O)[C@H]2O)c(=O)n1</smiles>

Fig. 4 Thiolysis and oxidation of pyrimidine nucleosides. a i. $\mathrm{H}_{2} \mathrm{~S}$ thiolysis of arabino-anhydrocytidine (3C) in water (pH 7) furnished $\beta$-arabino-cytidine (ara-4C; quant.) rapidly; ii. ara-4C then undergoes slow addition of $\mathrm{H}_{2} \mathrm{~S}$ to afford 4-thio- $\beta$-arabino-uridine (ara-7U). iii. $\mathrm{H}_{2} \mathrm{O}_{2}$ oxidation of ara-7U in water $(\mathrm{pH} 7)$ furnished arabino-uridine (ara-4U, 78\%). iv. Thiolysis of $\mathbf{3 C}$ in formamide yielded 2-thio- $\beta$-arabino-cytosine $(\boldsymbol{a r a}-\mathbf{6 C}, 31 \%), v$. $\mathrm{H}_{2} \mathrm{O}_{2}$ oxidation of ara$\mathbf{6 C}$ in water $(\mathrm{pH} 7)$ furnished $\mathbf{3 C}$ (quant.). b i-ii. $\mathrm{H}_{2} \mathrm{~S}$ thiolysis of ribo-anhydrocytidine ( $\alpha$-ribo-3C) in water ( $\left.\mathrm{pH} 7\right)$ furnished $\alpha$-ribo-cytidine ( $\alpha$-ribo-4C), which then undergoes addition of $\mathrm{H}_{2} \mathrm{~S}$ to afford 4-thio- $\alpha$-ribo-uridine ( $\alpha$-ribo-7U) in $91 \%$ combined conversion. iii. $\mathrm{H}_{2} \mathrm{O}_{2}$ oxidation of $\alpha$-ribo-7U in water ( $\mathrm{pH}$ 7) furnished $\alpha$-ribo-uridine ( $\alpha$-ribo-4U, 93\%). iv-v. Sutherland and co-workers reported the synthesis of 2 -thio- $\beta$-ribo-cytidine ( $\beta$-ribo-6C) by thiolysis of ribo-anhydrocytidine $\left(\alpha\right.$-ribo-3C) in formamide, followed by aqueous irradiation $(\lambda=254 \mathrm{~nm})^{35}$. vi. $\mathrm{H}_{2} \mathrm{O}_{2}$ oxidation of 2-thio- $\beta$-ribo-cytosine $(\beta$-ribo-6C) furnished canonical ribonucleoside $\beta$-cytidine ( $\beta$-ribo-4C, quant.) between $\mathrm{pH} 7$ and 9 . vii. Conversely, $\mathrm{H}_{2} \mathrm{O}_{2}$ oxidation of $\beta$-ribo-6C predominately furnished pyridimide 4-amino-pyrimidine-riboside $(\beta-$ ribo-9, $76 \%)$ at $\mathrm{pH}$ 3. viii. Due to the proximity of the nucleobase and $\mathrm{C2}^{\prime}$-hydroxyl moieties, $\mathrm{H}_{2} \mathrm{O}_{2}$ oxidation of $\alpha$-ribo-6C furnished $\alpha$-ribo-3C (quant.). ix-x. The sequential reaction of $\mathrm{H}_{2} \mathrm{~S}$ and $\mathrm{H}_{2} \mathrm{O}_{2}$ with $\beta$-ribo-4C in water (pH 7) furnished $\beta$-ribo-4U

triplet minimum, which would be sufficient to promote efficient photo-reduction. In principle, C-S bond homolysis (Fig. 3b) would be reversible, however tautomerisation of the intermediate C8-carbene to $\boldsymbol{a r a} \mathbf{- 4}$ would prevent reformation of $\boldsymbol{a r a - 5}$.

8-Mercaptopurine oxidation. Encouraged by the photochemical reduction of $\boldsymbol{a r a}-\mathbf{5 A}, \boldsymbol{a r a}-\mathbf{5 G}$ and $\boldsymbol{a r a}-\mathbf{5 I}$, we investigated how to increase the efficiency of purine reduction. Simplicity and prebiotic plausibility led us to consider hydrogen peroxide $\left(\mathrm{H}_{2} \mathrm{O}_{2}\right)$ in nucleobase reduction ${ }^{48-50}$. In our hands, the reported reaction of $\mathrm{H}_{2} \mathrm{O}_{2}$ and $\boldsymbol{a r a}-\mathbf{5 A}$ in acidic methanol ${ }^{36}$ gave ara-4A $(50 \%$ after $16 \mathrm{~h}$, Supplementary Fig. 17). Pleasingly, oxidative disproportionation of $\boldsymbol{a r a}-\mathbf{5 A}(50 \mathrm{mM})$ and $\boldsymbol{a r a}-\mathbf{5 I}(50 \mathrm{mM})$ under plausibly prebiotic conditions $\left(\mathrm{H}_{2} \mathrm{O}_{2}\right.$ (3 equiv), $\mathrm{pH} 7$, water, room temperature, $3 \mathrm{~h}$ ) afforded excellent conversion to $\boldsymbol{a r a}-\mathbf{4 A}(85 \%)$ and $\boldsymbol{a r a}-4 \mathrm{I}$ (90\%), respectively (Table 1 and Supplementary Figs. 18 and 19). Reaction of 1:1 ara-5A/ara-5I afforded ara-4A
(88\%) and ara-4I (91\%) (Supplementary Fig. 20). Next, we investigated the one-pot thiolysis/oxidation of 3G. Incubation of $3 \mathrm{G}(35 \mathrm{mM})$ with $\mathrm{H}_{2} \mathrm{~S}(714 \mathrm{mM})$ in water $\left(\mathrm{pH} 7,60^{\circ} \mathrm{C}, 7 \mathrm{~d}\right)$ then addition of $\mathrm{H}_{2} \mathrm{O}_{2}(375 \mu \mathrm{mol} ; \mathrm{pH} 7$, room temperature, $2 \mathrm{~h}$ ) furnished ara-4G (89\%) (Table 1 and Supplementary Fig. 21).

Pyrimidine thiolysis. Having demonstrated the efficient conversion of 3A, 3G and 3I to arabino-purines ara-4A, ara-4G and ara-4I, respectively, we next investigated the reactivity of pyrimidine precursor 3C under comparable conditions. Sutherland and co-workers reported that the reaction of a-ribo-anhydrocytidine (a-ribo-3C) with sodium hydrogen sulfide (NaHS) in formamide at $50{ }^{\circ} \mathrm{C}$ yields 2-thio- $\alpha$-cytidine (a-ribo-6C; $84 \%$; Fig. $4 \mathrm{~b} . i v)^{35}$. Similarly, upon submitting $3 \mathrm{C}$ to these conditions (NaHS, formamide, $50^{\circ} \mathrm{C}$ ), we observed conversion to ara-4C (24\%) and 2-thio-arabino-cytidine (ara-6C; 31\%) (Fig. 4a.iv; Supplementary Fig. 24). Conversely, incubation of 3C $(28 \mathrm{mM})$ 
with $\mathrm{H}_{2} \mathrm{~S}(0.14 \mathrm{M})$ in water $\left(\mathrm{pH} 7,60^{\circ} \mathrm{C}\right)$ yielded mostly ara- $4 \mathrm{C}$ (68\%) alongside some conversion of ara-4C to 4-thio-arabinouridine (ara-7U; 22\%) after 2 d (Fig. 4a.i-ii; Supplementary Fig. 25); $<5 \%$ ara-6C was observed. Further conversion of ara-4C to ara-7U was observed upon prolonged incubation (57:38 ara4C/ara-7U after 7 d; Fig. 4a.ii). Although in formamide the addition of NaHS to $\mathbf{3 C}$ furnishes ara-6C, in water rapid hydrolysis of $\mathbf{3 C}$ to ara-4C occurs, which subsequently undergoes slow nucleophilic substitution at the C4-carbon atom to furnish ara-7U.

The observed C4-thiolysis of ara-4C to ara-7U is not limited to the $\beta$-arabino-stereochemistry shown in Fig. 4a.ii. Submitting a-ribo-3C $(28 \mathrm{mM})$ to the same aqueous thiolysis conditions afforded $\alpha$-ribo-cytidine (a-ribo-4C, 52\%; Fig. 4b.ii) and 4-thio- $\alpha$ ribo-uridine (a-ribo-7U, 39\%; Fig. 4b.ix). Incubation of ara-4C, $\boldsymbol{\beta}$-ribo-4C and $\boldsymbol{\alpha}$-ribo-4C with $\mathrm{H}_{2} \mathrm{~S}(\mathrm{pH})$ led to partial (unoptimised) conversion to the 4-thiouridines $\operatorname{ara}-7 \mathrm{U}(7 \mathrm{~d}$, 40\%), $\boldsymbol{\beta}$-ribo-7U (7 d, 16\%) and $\boldsymbol{\alpha}$-ribo-7U (8 d, 63\%), respectively (Table 1), alongside recovered cytidine starting material [ara-4C (52\%), $\boldsymbol{\beta}$-ribo-4C (84\%) and $\boldsymbol{\alpha}$-ribo-4C (37\%)] (Supplementary Figs. 28-30). We were surprised to observe slower C4-thiolysis for the canonical trans-1', $2^{\prime}$-isomer ( $\beta$-ribo4C) than for the cis-1', $2^{\prime}$-isomers ( $\alpha$-ribo-4C and ara-4C). Simultaneous thiolysis of $1: 1$ ara-4C/ $\mathbf{\beta}$-ribo-4C verified this rate difference; after $7 \mathrm{~d}$ more ara-7U (52\%) than $\boldsymbol{\beta}$-ribo-7U (18\%) was observed (Supplementary Fig. 31), indicating the relative nucleobase/ $\mathrm{C2}^{\prime}-\mathrm{OH}$ orientation affects the rate of nucleophilic substitution at the distal C4-position of cytidine nucleotides. Finally, we incubated pyrimidine $\mathbf{3 C}$ and purine $\mathbf{3 A}$ (1:1) with $\mathrm{H}_{2} \mathrm{~S}\left(\mathrm{pH} 7,60^{\circ} \mathrm{C}, 7 \mathrm{~d}\right)$. Gratifyingly, ara-4C (70\%), ara-7U (30\%) and ara-5A (71\%) were cleanly furnished (Supplementary Fig. 27).

Pyrimidine oxidation. An efficient protocol to convert ara-7U to ara-4U would indicate that nucleosides ara-4C and ara-4U could be readily generated in comparable yields from thiolysis of 3C alongside conversion of $\mathbf{3 A} / \mathbf{3 G}$ to ara- $\mathbf{4 A} / \mathbf{a r a}-\mathbf{4 G}$, respectively. We envisioned that the thiocarbonyl moiety of ara-7U would be readily oxidised, however we suspected that oxidation would activate the pyrimidine nucleobase to hydrolysis (rather than reduction as observed for the purines). To test our hypothesis, we incubated ara-7U $(50 \mathrm{mM})$, $\boldsymbol{\beta}$-ribo- $7 \mathrm{U}(50 \mathrm{mM})$ and $\alpha$-ribo-7U $(50 \mathrm{mM})$ with $\mathrm{H}_{2} \mathrm{O}_{2}(0.15 \mathrm{M})$ in water $(\mathrm{pH} 7$, room temperature, $3.5 \mathrm{~h}$ ). Each reaction proceeded smoothly to give the respective uridine (ara-4U (78\%); $\boldsymbol{\beta}$-ribo-4U (78\%); a-ribo-4U (93\%); Table 1, Fig. 4 and Supplementary Figs. 38, 40-41), which validated our prediction. We next investigated thiolysis and selective hydrolysis in a one-pot reaction: $3 \mathrm{C}(35.7 \mathrm{mM})$ was incubated with $\mathrm{H}_{2} \mathrm{~S}(0.71 \mathrm{M})$ in water $\left(\mathrm{pH} 7,60^{\circ} \mathrm{C}, 7 \mathrm{~d}\right)$ and then $\mathrm{H}_{2} \mathrm{O}_{2}(375 \mu \mathrm{mol})$ was added. We observed concomitant formation of ara-4C (62\%) and ara-4U (25\%) (Supplementary Fig. 39).

The reactions of 2-thiocytidines ara-6C and a-ribo-6C with $\mathrm{H}_{2} \mathrm{O}_{2}(150 \mathrm{mM})$ in water $(\mathrm{pH} 7$, room temperature, $2 \mathrm{~h}$ ) cleanly regenerated anhydrocytidines 3C (82\%; Fig. 4a.v) and a-ribo-3C (80\%; Fig. 4b.viii), respectively, demonstrating a clear switch in reactivity relative to ara-7U due to the proximity of the $\mathrm{C} 2^{\prime}$ hydroxyl and thiocarbonyl moieties. Therefore, it is of note that canonical cytidine $\boldsymbol{\beta}$-ribo-6C has an anti-1', $2^{\prime}$-disposition, and we expected to observe hydrolysis during $\mathrm{H}_{2} \mathrm{O}_{2}$ oxidation of $\boldsymbol{\beta}$-ribo-6C. As expected, incubation of $\boldsymbol{\beta}$-ribo-6C (38.4 mM) with $\mathrm{H}_{2} \mathrm{O}_{2} \quad(232 \mathrm{mM})$ in phosphate buffer (pH 7-9, room temperature, $7 \mathrm{~h}$ ) afforded a quantitative conversion to $\boldsymbol{\beta}$-ribo-4C (Fig. 4b.vi; Supplementary Fig. 44). Interestingly, buffering the oxidation at $\mathrm{pH} 3$ with glycine afforded $\boldsymbol{\beta}$-ribo-4C (24\%) alongside 4-amino-pyrimidine-riboside ( $\beta$-ribo-9; 76\%;
Fig. 4b.vii; Supplementary Fig. 44). It is likely that cytidine protonation switches on C2-reduction by promoting access to the carbene intermediate required for reduction (Fig. 4b.vii) ${ }^{51}$. This hypothesis is supported by the simultaneous oxidation of ara-7U and $\boldsymbol{\beta}$-ribo-6C with $\mathrm{H}_{2} \mathrm{O}_{2}(\mathrm{pH} \quad 3$, glycine buffer, room temperature), which affords $\boldsymbol{\beta}$-ribo-4C (20\%) and $\boldsymbol{\beta}$-ribo-9 (72\%) from $\beta$-ribo-6C, but exclusively ara-4U (92\%) from ara7 U (Supplementary Fig. 45).

It is particularly interesting, with respect to the origins of life, that purine C8-reduction is facile and quantitative at neutral $\mathrm{pH}$, whereas pyrimidine $\mathrm{C} 2$-reduction is only observed at low $\mathrm{pH}$. Consequently, the reaction of $\boldsymbol{\beta}$-ribo- $6 \mathbf{C}$ with $\mathrm{H}_{2} \mathrm{O}_{2}$ at neutral $\mathrm{pH}$ furnishes canonical $\beta$-ribo-4C cleanly, but protects against, and even reverses, the formation of non-canonical ara-6C and a-ribo-6C. This provides a facile and selective method to convert $\boldsymbol{\beta}$-ribo-6C ${ }^{35}$ to canonical nucleoside $\boldsymbol{\beta}$-ribo-4C.

Concomitant purine and pyrimidine synthesis. To form information-rich ANA nucleic acid oligomers, all four Watson-Crick base-pairing nucleosides (ara-4A, ara-4C, ara-4G and ara-4U) need to accrue at the same time in the same environment, ideally from the same set of chemical reactions. We reacted 1:1:1 3C, 3A and 3G with $\mathrm{H}_{2} \mathrm{~S}\left(\mathrm{pH} 7,60^{\circ} \mathrm{C}\right.$; Supplementary Fig. 46), and after $7 \mathrm{~d}$ we observed ara-5A (65\%), ara5G (62\%), ara-4C (55\%) and ara-7U (35\%). Subsequent incubation with $\mathrm{H}_{2} \mathrm{O}_{2}(\mathrm{pH} 7$, room temperature, $2 \mathrm{~h}$ ) gave the desired Watson-Crick base-pairing products ara-4A (53\%), ara-4G (62\%), ara-4C (47\%) and ara-4U (35\%) without purification or isolation of intermediate products (Fig. 5). Thus, we achieved a plausibly prebiotic divergent synthesis of Watson-Crick basepairing nucleosides, and have marked ANA as a likely candidate for the nucleic acid of early evolution.

\section{Discussion}

Through the reaction of pyrimidine and purine precursors (3C and $3 \mathrm{~A}$, respectively), which can be accessed divergently from a single prebiotic substrate ${ }^{16,22}$, we have elucidated an efficient plausibly prebiotic method to simultaneously form arabino-pyrimidine and arabino-purine nucleosides. Both the purine and pyrimidine nucleosides are delivered with regiospecific glycosylation on a furanose-specific arabinose sugar moiety. The reaction of $\mathrm{H}_{2} \mathrm{~S}$ in water unlocks a purine-specific C8-reduction, by either UV irradiation or $\mathrm{H}_{2} \mathrm{O}_{2}$ oxidation; $\mathrm{H}_{2} \mathrm{O}_{2}$ oxidation is especially high yielding, however, UV irradiation caused selective

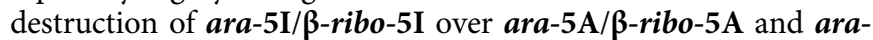
$\mathbf{5 G} / \boldsymbol{\beta}$-ribo-5G. The remarkable purity of the arabino-purines delivered by either route is highly encouraging, but the direct mechanism for canonical purine nucleobases selection by UV light provides a mechanism (based in the physical properties of the purine bases) for nucleobase selection prior to their incorporation into nucleic acid biopolymers at the origins of life.

In water $\mathrm{H}_{2} \mathrm{~S}$ also undergoes selective, but slow, addition to the cytidine C4-position, and-when coupled with oxidative hydrolysis-this provides a selective mechanism to convert cytidines to uridines. Importantly, $\mathrm{H}_{2} \mathrm{~S}$ did not convert $\mathrm{A} / \mathrm{G}$ to I/xanthine (X) demonstrating another remarkable, but essential, reactivity difference between the canonical nucleosides.

Although our focus has been on developing new chemistry, not on assessing geochemical boundary conditions, we note that the availability of $\mathrm{H}_{2} \mathrm{~S}$ and $\mathrm{H}_{2} \mathrm{O}_{2}$ are both plausibly prebiotic, and although their sequential reaction implicates different redox states this is not geochemically implausible. The entire planet is at redox disequilibrium, and layered redox gradients are geochemically common. Outgassing volatile compounds (from Earth's interior) are likely to have played a critical role in determining prebiotic 
a<smiles>[Y]c1nc([X])c2nc3n(c2n1)[C@@H]1O[C@H](CO)[C@@H](O)[C@H]1O3</smiles>

3A $\left(X=\mathrm{NH}_{2}, \mathrm{Y}=\mathrm{H}\right)$ 3G $\left(X=\mathrm{OH}, Y=\mathrm{NH}_{2}\right)$<smiles>[NH2+]=c1ccn2c(n1)O[C@@H]1[C@@H](O)[C@@H](CO)O[C@@H]12</smiles>

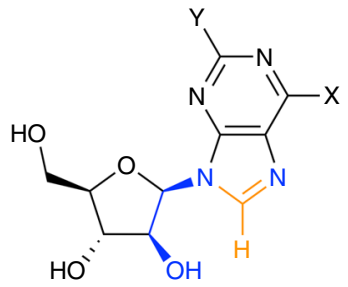

ara-4A $\left(X=\mathrm{NH}_{2}, \mathrm{Y}=\mathrm{H}\right)$ ara-4G $\left(\mathrm{X}=\mathrm{OH}, \mathrm{Y}=\mathrm{NH}_{2}\right)$

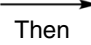

$\mathrm{H}_{2} \mathrm{O}_{2}$

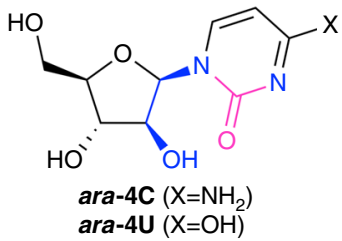

Fig. 5 Concomitant synthesis of four Watson-Crick base-pairing nucleosides. a One-pot reaction of an equimolar mixture of anhydroadenosine (3A), anhydroguanosine (3G) and anhydrocytidine (3C) with $\mathrm{H}_{2} \mathrm{~S}$ (2O equiv., $\mathrm{pH} 7$ ) in water, then $\mathrm{H}_{2} \mathrm{O}_{2}$ (20 equiv., $\mathrm{pH}$ 7) in water affords adenosine ara-4A (53\%), guanosine ara-4G (62\%), cytidine ara4C (47\%) and uridine ara-4U (35\%). The isourea moieties (blue) of the anhydropurines $(\mathbf{3 A} / \mathbf{G})$ and anhydropyrimidine (3C) were differentiated in situ to afford the canonical Watson-Crick base-pairing nucleobase moieties regiospecifically on a furanosyl-sugar scaffold, by reduction (orange) and hydrolysis (magenta), respectively. $\mathbf{b}^{1} \mathrm{H}$ NMR spectrum ( $600 \mathrm{MHz}, 9: 1 \mathrm{H}_{2} \mathrm{O} / \mathrm{D}_{2} \mathrm{O}, 25^{\circ} \mathrm{C}, \delta=5.5-7.0 \mathrm{ppm}$ ) showing the prebiotic synthesis of cytidine ara-4C, uridine ara-4U, adenosine ara-4A and guanosine ara-4G following the sequential addition of $\mathrm{H}_{2} \mathrm{~S}$ and $\mathrm{H}_{2} \mathrm{O}_{2}$ to anhydrocytidine $\mathbf{3 C}$, anhydroadenosine $\mathbf{3 A}$ and anhydroguanosine $\mathbf{3 G}$ in water. c ${ }^{1} \mathrm{H}$ NMR spectrum $\left(600 \mathrm{MHz}, 9: 1 \mathrm{H}_{2} \mathrm{O} / \mathrm{D}_{2} \mathrm{O}, 25^{\circ} \mathrm{C}, \delta=5.5-7.0\right.$ $\mathrm{ppm}$ ) of substrates anhydrocytidine $\mathbf{3 C}$, anhydroadenosine $\mathbf{3 A}$ and anhydroguanosine $\mathbf{3 G}$ in water

chemistry, and local redox environments (for example, due to volcanic outgassing, meteorite impacts, photo-oxidation and atmospheric water dissociation and hydrogen escape) are expected to provide significant variation from the global average ${ }^{52} \cdot \mathrm{H}_{2} \mathrm{~S}$ is produced through a distinct mechanism from $\mathrm{H}_{2} \mathrm{O}_{2}$, thus discrete redox zones could be readily established. The oxidation state of early Earth (Hadean) magmas are not well constrained, however, zircons that pre-date the known rock record suggest average oxygen fugacities may have been similar to the presentday conditions ${ }^{53}$. Accordingly, sulfide-leaching and $\mathrm{H}_{2} \mathrm{~S}$ outgassing would have occurred on the early Earth, with sulfur outgassing rates as high as $10^{11.5} \mathrm{~cm}^{-2} \mathrm{~s}^{-1}$ possible during major volcanogenic emplacement of basaltic plains ${ }^{54}$. Equally, photo-

dissociation generates $\mathrm{H}_{2} \mathrm{O}_{2}$ from water, and $\mathrm{H}_{2} \mathrm{O}_{2}$ is a likely key environmental oxidant prior to global oxidation ${ }^{37,50,55}$. It seems reasonable to suppose that distinct (redox) reactivity could be controlled by geochemical localisation and the different physicochemical processes that yield and accumulate feedstock molecules. Geochemical $\mathrm{H}_{2} \mathrm{~S}$ outgassing and atmospheric $\mathrm{H}_{2} \mathrm{O}_{2}$ production are well suited to this specific localisation. Moreover, it has recently been proposed that $\mathrm{H}_{2} \mathrm{O}_{2}$ can accumulate within ices in an anoxic atmosphere ${ }^{56}$. Melting $\mathrm{H}_{2} \mathrm{O}_{2}$-rich ices could augment $\mathrm{H}_{2} \mathrm{O}_{2}$ delivery into aqueous environments and, in principle, could help to provide a mechanism for the sequential delivery of $\mathrm{H}_{2} \mathrm{~S}$ and $\mathrm{H}_{2} \mathrm{O}_{2}$ into, for example, a flowing stream system or pool. Although the reactions of $\mathrm{H}_{2} \mathrm{~S}$ and $\mathrm{H}_{2} \mathrm{O}_{2}$ must occur in sequence to achieve purine reduction by the described oxidative mechanism, it is of note that the purine anhydronucleotides ( $3 \mathbf{A}$ and $\mathbf{3 G}$ ) were observed to be stable to $\mathrm{H}_{2} \mathrm{O}_{2}$ oxidation and the reduced purines (ara-4A and $\boldsymbol{a r a}-\mathbf{4 G}$ ) were observed to be stable to $\mathrm{H}_{2} \mathrm{~S}$ addition. Therefore, cycling material between these redox conditions (or across this redox gradient) is not considered to be problematic for this chemistry; indeed, a one-pot two-step $\mathrm{H}_{2} \mathrm{~S} / \mathrm{H}_{2} \mathrm{O}_{2}$ reduction has been demonstrated. Importantly, photochemical mercaptopurine reduction does not require two redox states, only $\mathrm{H}_{2} \mathrm{~S}$ and $\mathrm{UV}$ light (at $\lambda=$ 250-300 nm), both of which are expected to be in adequate (simultaneous) supply on the early Earth when sulfur outgassing rates are less than $10^{11.5} \mathrm{~cm}^{-2} \mathrm{~s}^{-1}$. The localised enhancement in $\mathrm{H}_{2} \mathrm{~S}$ can be achieved in surface hydrothermal systems with shallow water reservoirs (to allow UV penetration) ${ }^{54}$. In terms of the UV-light dose environment, we note that in the laboratory only six of the available sixteen lamps in the Rayonet irradiation chamber were employed, which corresponds to $39,000 \mathrm{erg} \mathrm{s}^{-1} \mathrm{~cm}^{-2}$ and $30,900 \mathrm{erg} \mathrm{s}^{-1} \mathrm{~cm}^{-2}$ at $\lambda=254 \mathrm{~nm}$ and $300 \mathrm{~nm}$, respectively. The integrated surface flux $(\lambda=200-300 \mathrm{~nm})$ delivered to the Earth by the early Sun is estimated to be about $2700 \mathrm{erg} \mathrm{s}^{-1} \mathrm{~cm}^{-2}$ (and within roughly an order of magnitude of the experimental apparatus), which provides ample flux to achieve the desired transformations and preserve prebiotic plausibility ${ }^{39}$. Crucially, the two distinct mercaptopurine 5 reductions demonstrate, not only the specific value of sulfur in prebiotic nucleoside synthesis, but also, more generally, the value of chemical redundancy. Two mechanisms for mercaptopurine reduction that operate under different conditions but both furnish the same purine products renders the overall transformation more robust, which may be especially important to consider in the context of the origins of life. Chemical redundancy is highly likely to improve pathway or network robustness, which may be an essential feature of sustained protometabolism in a (geo)chemically fluctuating environment ${ }^{3,18}$.

The different reactions observed between $\mathbf{3 C}$ and $\mathbf{3 A} / \mathbf{G}$ with $\mathrm{H}_{2} \mathrm{~S}$ is ideally suited to concomitant synthesis of the canonical nucleobases on preformed sugar scaffolds. Although the arabinostereochemistry is not found in extant genetics, it is highly plausible that ANA could have been a precursor to RNA in early life or that early co-evolution of mixed RNA/ANA systems could have been superseded by RNA/DNA systems. The simplicity and efficiency of the synthesis of $A, C, G$ and $U$ arabinosides indicates that further investigations into the synthesis of $\mathbf{3 G}$ and the potential for ANA and RNA co-evolution are both warranted.

\section{Methods}

General procedure A. Thiolysis. Sodium hydrosulfide or disodium sulfide nonahydrate (20 equiv.) was dissolved in $\mathrm{H}_{2} \mathrm{O} / \mathrm{D}_{2} \mathrm{O}(9: 1)$ at $\mathrm{pH}$ 7. Nucleoside(s) (1 equiv.) was added and incubated at $\mathrm{pH} 7$ and $60^{\circ} \mathrm{C}$. The reaction progress was monitored periodically by NMR spectroscopy. Upon completion, the reaction was cooled to room temperature and sparged of hydrogen sulfide with nitrogen or argon gas. The solution was adjusted to $\mathrm{pH} 6.5$ and analysed by NMR spectroscopy. 
General procedure B. UV irradiation. A degassed aqueous solution of nucleoside (s) $(2.00 \mathrm{mM}, \mathrm{pH}$ 6.5) was irradiated in a Rayonet reactor (SNE Ultraviolet Co.) housing six RPR-2537A or RPR-3000A lamps (with principal emission at $\lambda=254 \mathrm{~nm}$ and $\lambda=300 \mathrm{~nm}$, respectively) at $38^{\circ} \mathrm{C}$ under an argon atmosphere. After irradiation, the reaction was allowed to cool to room temperature, and then lyophilised. The lyophilisate was dissolved in $\mathrm{D}_{2} \mathrm{O}(500 \mu \mathrm{L})$ and analysed by NMR spectroscopy. A solution of potassium hydrogen phthalate $(0.100 \mathrm{M}, 50.0 \mu \mathrm{L}, 5.00$ $\mu \mathrm{mol}$ in $\mathrm{D}_{2} \mathrm{O}$ ) was added as an internal NMR standard and NMR spectra were reacquired.

General procedure C. Nucleoside oxidation. Nucleoside(s) (1.0 equiv., $50 \mathrm{mM}$ ) and potassium hydrogen phthalate (0.2 equiv.) were dissolved in $\mathrm{H}_{2} \mathrm{O} / \mathrm{D}_{2} \mathrm{O}(9: 1)$ or buffer solution. The $\mathrm{pH}$ of the solution was adjusted to $\mathrm{pH} 7$ and NMR spectra were acquired. Hydrogen peroxide $\left(30 \% \mathrm{w} / \mathrm{w}\right.$ solution in $\left.\mathrm{H}_{2} \mathrm{O}\right)$ was added and NMR spectra were periodically acquired at $\mathrm{pH} 7$.

Computational methods. The vertical excitation energies, excited state geometries and excited state harmonic vibrational frequencies of ara-5I, ara-5A and ara-5G were computed by using the algebraic diagrammatic construction to the secondorder method $[\mathrm{ADC}(2)]^{40,41,57}$, and the cc-pVTZ basis set. The MP2/cc-pVTZ method was used to optimise the corresponding ground-state geometries. Spin-orbit coupling matrix elements were calculated at the CASPT2/SA-CASSCF $(10,9) / c c-p V T Z-D K ~ l e v e l{ }^{58}$, including the second-order Douglas-Kroll-Hess transformation to account for scalar relativistic effects. Calculated ESA spectra were obtained by convolution of vertical excitation energies and oscillator strengths with normalised Gaussian functions $(0.20 \mathrm{eV}$ half-width). Vertical excitation energies necessary for the ESA spectra were calculated from the corresponding excited state minima and 11 excitations were taken into account in each case. ESA cross-sections were generated using the GaussSum programme ${ }^{59}$. Minimum-energy crossing point (MECPs) geometries were optimised with the in-house implementation of the method proposed by Levine, Coe and Martine $z^{60}$. The energies and gradients in electronically excited states were computed at the $\operatorname{ADC}(2)$ level, whereas the corresponding properties for electronic ground states were obtained at the MP2 level in the MECP geometry optimisations ${ }^{41}$. The MECP optimisation steps were performed with the Broyden-Fletcher-Goldfarb-Shanno quasi-Newton scheme available in the internal optimiser of Turbomole $7.1^{61}$. All ADC(2) and MP2 calculations were performed with Turbomole 7.1, and Molcas $8.0^{62}$ was employed for all the CASPT2/SA-CASSCF calculations. Graphical representations of the molecular geometries and orbitals were generated with IboView ${ }^{63}$.

Femtosecond transient absorption spectroscopy. FTAS experiments were performed with a Solstice Ace pulsed laser system (Spectra-Physics, Newport Co.), which produces $97 \mathrm{fs}$ pulses at $\lambda=800 \mathrm{~nm}$. To generate the white light continuum probe pulses $(\lambda=320-700 \mathrm{~nm})$ in the Helios-Fire spectrometer (Ultrafast Systems, LLC), a fraction of the fundamental beam was focused on a thin $\mathrm{CaF}_{2}$ crystal. The $\lambda=290 \mathrm{~nm}$ excitation (pump) pulses were generated by passing the remainder of the fundamental beam through an optical parametric amplifier (TOPAS, Light Conversion, Ltd.). FTAS experiments of aqueous samples of ara-5A $(4 \mathrm{mM}, \mathrm{pH}$ 7.4) and ara-5I ( $4 \mathrm{mM}, \mathrm{pH} 7.4)$ were performed in $2 \mathrm{~mm}$ optical path length quartz cuvettes (Starna Cells, Inc.). LabView Surface Xplorer software (Ultrafast Systems, LLC) was used to reduce the FTAS data, apply corrections for group velocity dispersion of the white light probe, and extract and analyse spectra and their time dependence.

X-ray diffraction. All diffraction data were collected by using a four-circle Agilent SuperNova (Dual Source) single crystal X-ray diffractometer with a micro-focus $\mathrm{CuK}_{\alpha} \mathrm{X}$-ray beam $(\lambda=1.54184 \AA)$ and an Atlas CCD detector. The crystal temperature was controlled by using an Oxford Instruments Cryojet5. Unit cell determination, data reduction and analytical numeric absorption correction using a multifaceted crystal were carried out using the CrysAlisPro programme ${ }^{64}$. The crystal structures were solved with the ShelXS programme and refined by least squares on the basis of $\mathrm{F}^{2}$ with the ShelXL programme ${ }^{65}$. All non-hydrogen atoms were refined anisotropically by the full-matrix least-squares method. Hydrogen atoms affiliated with oxygen and nitrogen atoms were refined isotropically in positions identified by the difference Fourier map, or in geometrically constrained positions. Hydrogen atoms associated with carbon atoms were refined isotropically in geometrically constrained positions.

\section{Data availability}

The authors declare that data supporting the findings of this study are available within the paper and its Supplementary Information files and figures. X-ray crystallographic data were deposited at the Cambridge Crystallographic Data Centre (CCDC) under the following CCDC deposition numbers: 1586272 (ara-5A, Supplementary Fig. 120) and 1836032 (3',3-anhydro-guanosine (12); the high $\mathrm{pH}$ isomerisation product of 3G, Supplementary Fig. 121). These can be obtained free of charge from CCDC via https:// www.ccdc.cam.ac.uk/structures/.
Received: 25 May 2018 Accepted: 31 August 2018

Published online: 04 October 2018

\section{References}

1. Gilbert, W. The RNA world. Nature 319, 618 (1986).

2. Orgel, L. E. Prebiotic chemistry and the origin of the RNA world. Crit. Rev. Biochem. Mol. Biol. 39, 99-123 (2004).

3. Islam, S. \& Powner, M. W. Prebiotic systems chemistry: complexity overcoming clutter. Chem 2, 470-501 (2017).

4. Eschenmoser, A. The search for the chemistry of life's origin. Tetrahedron 63 12821-12844 (2007).

5. Anastasi, C. et al. RNA: prebiotic product, or biotic invention? Chem. Biodivers. 4, 721-739 (2007).

6. Taylor, A. I. et al. Catalysts from synthetic genetic polymers. Nature 518, 427-430 (2015)

7. Fuller, W. D., Sanchez, R. A. \& Orgel, L. E. Studies in prebiotic synthesis. VI. Synthesis of purine nucleosides. J. Mol. Biol. 67, 25-33 (1972).

8. Joyce, G. F., Schwartz, A. W., Miller, S. L. \& Orgel, L. E. The case for an ancestral genetic system involving simple analogues of the nucleotides. Proc. Natl Acad. Sci. USA 84, 4398-4402 (1987).

9. Eschenmoser, A. \& Loewenthal, E. Chemistry of potentially prebiological natural products. Chem. Soc. Rev. 21, 1-16 (1992).

10. Böhler, C., Nielsen, P. E. \& Orgel, L. E. Template switching between PNA and RNA oligonucleotides. Nature 376, 578-581 (1995).

11. Eschenmoser, A. Chemical etiology of nucleic acid structure. Science 284, 2118-2124 (1999).

12. Nielsen, P. E. Peptide nucleic acid. A molecule with two identities. Acc. Chem. Res. 32, 624-630 (1999).

13. Schöning, K.-U. et al. Chemical etiology of nucleic acid structure: the athreofuranosyl- $\left(3^{\prime} \rightarrow 2\right)$ oligonucleotide system. Science 290, 1347-1351 (2000).

14. Orgel, L. E. Prebiotic adenine revisited: eutectics and photochemistry. Orig. Life Evol. Biosph. 34, 361-369 (2004).

15. Zhang, L., Peritz, A. \& Meggers, E. A simple glycol nucleic acid. J. Am. Chem. Soc. 127, 4174-4175 (2005).

16. Powner, M. W., Gerland, B. \& Sutherland, J. D. Synthesis of activated pyrimidine ribonucleotides in prebiotically plausible conditions. Nature 459 , 239-242 (2009).

17. Sutherland, J. D. Ribonucleotides. Cold Spring Harb. Perspect. Biol. 2, a005439 (2010).

18. Powner, M. W. \& Sutherland, J. D. Prebiotic chemistry: a new modus operandi. Philos. Trans. R. Soc. B Biol. Sci. 366, 2870-2877 (2011).

19. Rios, A. C. \& Tor, Y. On the origin of the canonical nucleobases: an assessment of selection pressures across chemical and early biological evolution. Isr. J. Chem. 53, 469-483 (2013).

20. Hud, N. V. et al. The origin of RNA and "my grandfather's axe". Chem. Biol. 20, 466-474 (2013)

21. Becker, S. et al. A high-yielding, strictly regioselective prebiotic purine nucleoside formation pathway. Science 352, 833-836 (2016).

22. Stairs, S. et al. Divergent prebiotic synthesis of pyrimidine and 8-oxo-purine ribonucleotides. Nat. Commun. 8, 15270 (2017).

23. Kim, H.-J. \& Benner, S. A. Prebiotic stereoselective synthesis of purine and noncanonical pyrimidine nucleotide from nucleobases and phosphorylated carbohydrates. Proc. Natl Acad. Sci. USA 114, 11315-11320 (2017).

24. Fialho, D. M. et al. Glycosylation of a model proto-RNA nucleobase with non-ribose sugars: implications for the prebiotic synthesis of nucleosides. Org. Biomol. Chem. 16, 1263-1271 (2018).

25. Martín-Pintado, N. et al. The solution structure of double helical arabino nucleic acids (ANA and 2'F-ANA): effect of arabinoses in duplex-hairpin interconversion. Nucleic Acids Res. 40, 9329-9339 (2012).

26. Noronha, A. M. et al. Synthesis and biophysical properties of arabinonucleic acids (ANA): circular dichroic spectra, melting temperatures, and ribonuclease $\mathrm{H}$ susceptibility of ANA.RNA hybrid duplexes. Biochemistry 39 , 7050-7062 (2000).

27. Li, F. et al. 2'-Fluoroarabino- and arabinonucleic acid show different conformations, resulting in deviating RNA affinities and processing of their heteroduplexes with RNA by RNase H. Biochemistry 45, 4141-4152 (2006).

28. Pinheiro, V. B. et al. Synthetic genetic polymers capable of heredity and evolution. Science 336, 341-344 (2012).

29. Van Nguyen, K. \& Burrows, C. J. A prebiotic role for 8-oxoguanosine as a flavin mimic in pyrimidine dimer photorepair. J. Am. Chem. Soc. 133, 14586-14589 (2011).

30. Leman, L., Orgel, L. \& Ghadiri, M. R. Carbonyl sulfide-mediated prebiotic formation of peptides. Science 306, 283-286 (2004).

31. Bowler, F. R. et al. Prebiotically plausible oligoribonucleotide ligation facilitated by chemoselective acetylation. Nat. Chem. 5, 383-389 (2013). 
32. Heuberger, B. D., Pal, A., Del Frate, F., Topkar, V. V. \& Szostak, J. W. Replacing uridine with 2-thiouridine enhances the rate and fidelity of nonenzymatic RNA primer extension. J. Am. Chem. Soc. 137, 2769-2775 (2015).

33. Patel, B. H., Percivalle, C., Ritson, D. J., Duffy, C. D. \& Sutherland, J. D. Common origins of RNA, protein and lipid precursors in a cyanosulfidic protometabolism. Nat. Chem. 7, 301-307 (2015).

34. Islam, S., Bucar, D.-K. \& Powner, M. W. Prebiotic selection and assembly of proteinogenic amino acids and natural nucleotides from complex mixtures. Nat. Chem. 9, 584-589 (2017).

35. $\mathrm{Xu}$, J. et al. A prebiotically plausible synthesis of pyrimidine $\beta$-ribonucleosides and their phosphate derivatives involving photoanomerization. Nat. Chem. 9 , 303-309 (2017).

36. Ikehara, M. \& Ogiso, Y. Studies of nucleosides and nucleotides-LIV. Purine cyclonucleosides-19. Further investigations on the cleavage of the $8,2^{\prime}$-Oanhydro linkage. A new synthesis of $9-\beta$-D-arabinofuranosyladenine. Tetrahedron 28, 3695-3704 (1972).

37. Kasting, J. F. Earth's early atmosphere. Science 259, 920-926 (1993).

38. Ribas, I. et al. Evolution of the solar activity over time and effects on planetary atmospheres. II. $\kappa-1$ Ceti, an analog of the sun when life arose on earth. Astrophys. J. 714, 384-395 (2010).

39. Ranjan, S. \& Sasselov, D. D. Influence of the UV environment on the synthesis of prebiotic molecules. Astrobiology 16, 1-80 (2016).

40. Dreuw, A. \& Wormit, M. The algebraic diagrammatic construction scheme for the polarization propagator for the calculation of excited states. Wiley Interdiscip. Rev. Comput. Mol. Sci. 5, 82-95 (2015).

41. Hättig, C. Structure optimizations for excited states with correlated secondorder methods: CC2 and ADC(2). Adv. Quantum Chem. 50, 37-60 (2005).

42. Martínez-Fernández, L., Corral, I., Granucci, G. \& Persico, M. Competing ultrafast intersystem crossing and internal conversion: a time resolved picture for the deactivation of 6-thioguanine. Chem. Sci. 5, 1336 (2014).

43. Pollum, M. \& Crespo-Hernández, C. E. Communication: the dark singlet state as a doorway state in the ultrafast and efficient intersystem crossing dynamics in 2-thiothymine and 2-thiouracil. J. Chem. Phys. 140, 071101 (2014).

44. Taras-Goślińska, K., Burdziński, G. \& Wenska, G. Relaxation of the T1excited state of 2-thiothymine, its riboside and deoxyriboside-enhanced nonradiative decay rate induced by sugar substituent. J. Photochem. Photobiol. A Chem. 275, 89-95 (2014).

45. Mai, S. et al. The origin of efficient triplet state population in sulfursubstituted nucleobases. Nat. Commun. 7, 1-8 (2016).

46. Bai, S. \& Barbatti, M. On the decay of the triplet state of thionucleobases. Phys. Chem. Chem. Phys. 19, 12674-12682 (2017).

47. El-Sayed, M. A. Spin-orbit coupling and the radiationless processes in nitrogen heterocyclics. J. Chem. Phys. 38, 2834-2838 (1963).

48. Fernández-García, C., Grefenstette, N. M. \& Powner, M. W. Prebiotic synthesis of aminooxazoline-5' - phosphates in water by oxidative phosphorylation. Chem. Commun. 53, 4919-4921 (2017).

49. Coggins, A. J. \& Powner, M. W. Prebiotic synthesis of phosphoenol pyruvate by a-phosphorylation-controlled triose glycolysis. Nat. Chem. 9, 310-317 (2016).

50. Kasting, J. F., Holland, H. D. \& Pinto, J. P. Oxidant abundances in rainwater and the evolution of atmospheric oxygen. J. Geophys. Res. 90, 10497-10510 (1985).

51. Sochacka, E., Bartos, P., Kraszewska, K. \& Nawrot, B. Desulfuration of 2thiouridine with hydrogen peroxide in the physiological pH range 6.6-7.6 is $\mathrm{pH}$-dependent and results in two distinct products. Bioorg. Med. Chem. Lett. 23, 5803-5805 (2013).

52. Hazen, R. M. Paleomineralogy of the hadean eon: a preliminary species list. Am. J. Sci. 313, 807-843 (2013).

53. Trail, D., Watson, E. B. \& Tailby, N. D. The oxidation state of hadean magmas and implications for early earth's atmosphere. Nature 480, 79-82 (2011).

54. Ranjan, S., Todd, Z. R., Sutherland, J. D. \& Sasselov, D. D. Sulfidic anion concentrations on early earth for surficial origins-of-life chemistry. Preprint at https://arxiv.org/abs/1801.07725 (2018).

55. Catling, D. C. \& Kasting, J. F. Atmospheric Evolution on Inhabited and Lifeless Worlds (Cambridge University Press, Cambridge, 2017).

56. Liang, M.-C., Hartman, H., Kopp, R. E., Kirschvink, J. L. \& Yung, Y. L. Production of hydrogen peroxide in the atmosphere of a snowball earth and the origin of oxygenic photosynthesis. Proc. Natl Acad. Sci. USA 103, 18896-18899 (2006).
57. Trofimov, A. B. \& Schirmer, J. An efficient polarization propagator approach to valence electron excitation spectra. J. Phys. B At. Mol. Opt. Phys. 28, 2299-2324 (1995)

58. Finley, J., Malmqvist, P.-Å., Roos, B. O. \& Serrano-Andrés, L. The multi-state CASPT2 method. Chem. Phys. Lett. 288, 299-306 (1998).

59. O’boyle, N. M., Tenderholt, A. L. \& Langner, K. M. cclib: A library for package-independent computational chemistry algorithms. J. Comput. Chem. 29, 839-845 (2008).

60. Levine, B. G., Coe, J. D. \& Martínez, T. J. Optimizing conical intersections without derivative coupling vectors: application to multistate multireference second-order perturbation theory (MS-CASPT2). J. Phys. Chem. B 112, 405-413 (2008).

61. Ahlrichs, R., Bär, M., Häser, M., Horn, H. \& Kölmel, C. Electronic structure calculations on workstation computers: the program system turbomole. Chem. Phys. Lett. 162, 165-169 (1989).

62. Aquilante, F. et al. Molcas 8: new capabilities for multiconfigurational quantum chemical calculations across the periodic table. J. Comput. Chem. 37, 506-541 (2016).

63. Knizia, G. \& Klein, J. E. M. N. Electron flow in reaction mechanisms-revealed from first principles. Angew. Chem. Int. Ed. 54, 5518-5522 (2015).

64. CrysAllisPro. Oxford Diffraction (Agilent Technologies, Inc., Yarnton, 2014).

65. Sheldrick, G. M., IUCr. A short history of SHELIX. Acta Crystallogr. Sect. A Found. Crystallogr. 64, 112-122 (2008).

\section{Acknowledgements}

This work was supported in part by the Simons Foundation (318881 to M.W.P., 494188 to R.S. and 290360 to D.D.S.) and the Engineering and Physical Sciences Research Council (EP/K004980/1 to M.W.P.). The authors thank Dr. K. Karu for assistance with mass spectrometry and Dr. A.E. Aliev for assistance with NMR spectroscopy. Z.R.T. and D.D.S. would like to thank D. Bucher, and to acknowledge the Harvard Origins of Life Initiative.

\section{Author contributions}

M.W.P. conceived the research. M.W.P. and S.J.R. designed and analysed the experiments. S.J.R. and S.S. conducted the experiments. D.K.B. performed the crystallographic analyses. J.S. oversaw the theoretical work, which R.S. carried out. Z.R.T. and D.D.S. performed and analysed the femtosecond transient absorption spectroscopy. M.W.P., R. S. and S.J.R. wrote the paper.

\section{Additional information}

Supplementary Information accompanies this paper at https://doi.org/10.1038/s41467018-06374-z.

Competing interests: The authors declare no competing interests.

Reprints and permission information is available online at http://npg.nature.com/ reprintsandpermissions/

Publisher's note: Springer Nature remains neutral with regard to jurisdictional claims in published maps and institutional affiliations.

Open Access This article is licensed under a Creative Commons Attribution 4.0 International License, which permits use, sharing, adaptation, distribution and reproduction in any medium or format, as long as you give appropriate credit to the original author(s) and the source, provide a link to the Creative Commons license, and indicate if changes were made. The images or other third party material in this article are included in the article's Creative Commons license, unless indicated otherwise in a credit line to the material. If material is not included in the article's Creative Commons license and your intended use is not permitted by statutory regulation or exceeds the permitted use, you will need to obtain permission directly from the copyright holder. To view a copy of this license, visit http://creativecommons.org/ licenses/by/4.0/.

(C) The Author(s) 2018 